\title{
Entropy rate defined by internal wave scattering in long-range propagation
}

\author{
Andrey K Morozova) \\ Department of Applied Ocean Physics and Engineering, Woods Hole Oceanographic Institution, \\ MS\# 09, Woods Hole, Massachusetts 02543, USA \\ John A. Colosi \\ Department of Oceanography Graduate School of Engineering and Applied Sciences, Naval Postgraduate \\ School, 833 Dyer Road, Monterey, California 93943, USA
}

(Received 4 September 2014; revised 20 July 2015; accepted 3 August 2015; published online 8 September 2015)

The reduction of information capacity of the ocean sound channel due to scattering by internal waves is a potential problem for acoustic communication, navigation, and remote sensing over long ranges. In spite of recent progress in research on acoustic signal scattering by random internal waves and the fact that random internal waves are ubiquitous in the world oceans, there is no clear understanding of how these waves influence data communication performance. The entropy decrease resulting from scattering by internal waves is an important measure of information loss. Here a rigorous calculation of the entropy is carried out using second moment transport theory equations with random sound-speed perturbations obeying the Garrett-Munk internal-wave model. It is shown that full-wave rate of entropy is of the same order of magnitude as the Kolmogorov-Sinai entropy and Lyapunov exponents for the relevant ray trajectories. The correspondence between full-wave and ray entropies suggests a correspondence between full-wave scattering and ray chaos near statistical saturation. The relatively small level of entropy rate during propagation through the random internal-wave field shows that scattering by internal waves is likely not an essential limitation for data rate and channel capacity.

(C) 2015 Acoustical Society of America. [http://dx.doi.org/10.1121/1.4928617]

[TFD]

Pages: $1353-1364$

\section{SCIENTIFIC OBJECTIVE DETERMINATION, MAIN RESULTS}

Scattering by small-scale ocean processes such as internal waves can impose limitations on ocean acoustic communications, navigation, remote sensing, and matched-field processing. The stochastic properties of the sound field scattered by internal waves are poorly understood in long-range propagation as conditions of saturation are established. ${ }^{1-9}$ Early studies utilizing ray methods to understand scattering processes in long-range acoustic propagation suggested there is an exponential sensitivity to initial conditions and a rapid growth of acoustic field complexity within a scale of a few hundred kilometers. ${ }^{1,2}$ Later examination of the phenomenon of ocean acoustic ray chaos with its manifestations in the wave field has shown that in the presence of a waveguide and realistic ocean sound-speed fluctuations the complexity of ocean acoustical beams do not expand exponentially or explosively but rather they expand diffusively. ${ }^{10}$ Shannon entropy is a natural measure of acoustical beam complexity, informational losses in long-range propagation, and estimation of communications channel capacity. Entropy evolution in a random ocean using Monte Carlo simulation within the parabolic equation approximation of sound propagation has been studied. ${ }^{10}$ As result of that simulation, for the first time,

\footnotetext{
a) Also at Teledyne TWR, 49 Edgerton Drive, N. Falmouth, Massachusetts 02536. Electronic mail: amorozov@teledyne.com
}

a close connection was found between Shannon entropy of a wave field and the Lyapunov exponent of ray chaos. The present work continues that investigation on a more rigorous theoretical foundation. The problem is solved using stochastic differential equations for the second-order statistical moments of the acoustic field ${ }^{7,8,11}$ and an equation for a Shannon entropy evolution is derived. The scattering of a narrow weakly divergent sound beam ${ }^{12}$ by random ocean structure is considered in the coupled-mode approximation. ${ }^{11}$ The random index of refraction is described using the Garrett-Munk internal-wave spectrum. ${ }^{3}$ The results are obtained in good agreement with the original research. ${ }^{10} \mathrm{We}$ consider the Shannon entropy of vertical profiles of the beam complex envelope as a function of range in a close connection between the instability of the ray equations (i.e., Lyapunov exponent) and the Kolmogorov-Sinai (K-S) entropy. ${ }^{2}$ The results show that the rate of change of Shannon entropy, for some idealized dynamical systems, is closely related to the K-S entropy over an intermediate regime of time. Thus the rate of Shannon entropy for our finitefrequency numerical simulations can be directly compared to ray simulation results of average finite-range Lyapunov exponents as a measure of K-S entropy.

In this study, it is found that acoustic beams in an ocean waveguide with realistic sound-speed perturbations caused by internal waves after a startup transient process expand diffusively with the entropy growing linearly with range. The entropy rate obtained in the simulation depends upon 
the emitting angle and has an order of magnitude Lyapunov exponent. This Shannon entropy rate is decreasing and converging to the value of Lyapunov exponent with frequency increasing. Specifically, the rate of change of Shannon entropy in this linear regime for frequency higher $300 \mathrm{~Hz}$ at the channel axis is computed to be approximately $0.02 \mathrm{bit} / \mathrm{km}$ and at the higher angle beam emitted from the depth $2000 \mathrm{~m}$ to be around $0.01 \mathrm{bit} / \mathrm{km}$. These values are shown to be close to $\mathrm{K}-\mathrm{S}$ entropy, computed for the same conditions in ray approximation and related to ray chaos. Clearly, the wave field entropy cannot grow indefinitely as this system is bounded by the ocean surface and bottom. If the waveguide is lossless, the entropy should stabilize to a level equivalent to equipartition, a maximum entropy state. The entropy will grow until acoustic energy fills the entire water column at which time the entropy should stabilize. The diffusive beam spreading and the linear rate of Shannon entropy is decreasing and converging to $\mathrm{K}-\mathrm{S}$ entropy when frequency is increasing. These results suggest a finite-frequency manifestation of ray chaos after the establishment of saturation. In conclusion, it will be shown that the small level of entropy rate caused by scattering by internal waves is not an essential limitation for data rate and channel capacity.

\section{STOCHASTIC DIFFERENTIAL EQUATIONS FOR SECOND ORDER MOMENTUMS OF RANDOM FIELD}

The following is a brief description of stochastic differential equations basics that have been previously published but are included here for completeness ${ }^{11}$ (see the Appendix).

Let us assume that the ocean sound speed $C(x, z)$ consists of an average sound-speed profile $\bar{C}(x, z)$, dependent only on the depth coordinate $z$, and a perturbation fluctuation $\delta c(x, z)$. Considering the acoustical pressure in a form,

$$
p(x, z)=\Psi(x, z) \exp \left[i\left(\kappa_{0} x-\omega t\right)\right] .
$$

The parabolic differential equation for the wave function $\Psi(x, z)$ has the form [Eq. (1)]

$$
-2 i \kappa_{0} \frac{\partial \Psi}{\partial x}=-\frac{\partial^{2} \Psi}{\partial z^{2}}+\left(\kappa_{0}^{2}-\kappa^{2}(x, z)\right) \Psi
$$

where

$$
\kappa=\omega / c(x, z), \kappa_{0}=\omega / c_{0} .
$$

Expansion of the wave-function in terms of the unperturbed modes $\varphi_{n}(z)$ has the form,

$$
\Psi(x, z)=\sum_{m=1}^{N} A_{m}(x) \varphi_{n}(z)
$$

where

$$
\left[\frac{d^{2}}{d z^{2}}+\bar{\kappa}^{2}(z)-\kappa_{n}^{2}\right] \varphi_{n}=0 ; \Psi(0, z)=\Psi_{0}(z) .
$$

The stochastic amplitudes obey the system of ordinary differential equations

$$
\frac{\partial A_{n}}{\partial x}=i \frac{\kappa_{n}^{2}-\kappa_{0}^{2}}{2 \kappa_{0}} A_{n}-i \sum_{m=1}^{N} \rho_{m n} A
$$

with the stochastic stationary mode coupling matrix

$$
\rho_{m n}(x)=k_{0} \int_{0}^{D} \frac{\delta c(x, z)}{c_{0}} \varphi_{n}(z) \varphi_{m}(z) d z,
$$

where $D$ is the ocean depth; $\kappa_{0}=\omega / c_{0}$.

After removing deterministic oscillations, the interaction representation of the equation takes the form,

$$
\frac{\partial u_{n}}{\partial x}=-i \sum_{m=1}^{N} \exp \left(-i k_{m} x\right) \rho_{m n} \exp \left(i k_{n} x\right) u_{m},
$$

where

$$
u_{n}=A_{n} \exp \left(-i k_{n} x\right) ; k_{n}=\frac{\kappa_{n}^{2}-\kappa_{0}^{2}}{2 k_{0}},
$$

or in a matrix form,

$$
\frac{\partial U}{\partial x}=i M U
$$

where

$$
\begin{aligned}
& U=\left\{A_{n} \exp \left(-i k_{n} x\right)\right\}, \\
& U(x=0)=U_{0}, M(x)=\left\{-e^{-i k_{m} x} \rho_{m n}(x) e^{i k_{n} x}\right\} .
\end{aligned}
$$

$M$ is a random square symmetrical matrix. The stochastic properties of $\mathrm{M}$ are completely defined by statistics of its random coefficients $-e^{-i k_{m} x} \rho_{m n}(x) e^{i k_{n} x}$ related to random sound velocity fluctuations $\delta c(x, z)$.

The stochastic equation ${ }^{13}$ for the correlation matrix $\langle\gamma\rangle=\left\langle U U^{* T}\right\rangle$, derived in the Appendix has a form,

$$
\begin{aligned}
\frac{\partial\langle\gamma\rangle}{\partial x}= & -\int_{0}^{\infty}\langle M(x) M(x-\xi)\rangle d \xi\langle\gamma\rangle \\
& -\langle\gamma\rangle \int_{0}^{\infty}\left\langle M^{*}(x) M^{*}(x-\xi)\right\rangle^{T} d \xi \\
& +\int_{0}^{\infty}\left\langle M(x-\xi)\langle\gamma\rangle(x) M^{* T}(x)\right\rangle d \xi \\
& +\int_{0}^{\infty}\left\langle M(x)\langle\gamma\rangle(x) M^{* T}(x-\xi)\right\rangle d \xi
\end{aligned}
$$

We are considering a stationary stochastic mode coupling coefficients with the cross-correlation function, which in accordance with a corollary of the Wiener-Khintchin theorem, is the inverse Fourier transformation of the crosspower spectral density function,

$$
\left\langle\rho_{m n}(x) \rho_{k l}^{*}(x-\xi)\right\rangle=\int_{-\infty}^{\infty} d k\left\langle\hat{\rho}_{m n}(k) \hat{\rho}_{k l}^{*}(k)\right\rangle e^{-i k \xi} .
$$


The correlation matrix $\left\langle\hat{\rho}_{m n}(k) \hat{\rho}_{k l}^{*}(k)\right\rangle$ defines the statistics of random matrix $M(x)=\left\{-e^{-i k_{m} x} \rho_{m n}(x) e^{i k_{n} x}\right\}$ and can be calculated from internal wave model (Colosi and Brown $\left.{ }^{3}\right)$. The specific model parameters are: number of internal wave modes $-N_{J}=50, \varsigma_{0}=7.3 \mathrm{~m}$,

$$
\left\langle\hat{\rho}_{m n}\left(k_{x}\right) \hat{\rho}_{k l}^{*}\left(k_{x}\right)\right\rangle=\sum_{J=1}^{N_{J}}\left\langle\left|a_{J}\right|^{2}\right\rangle\left\langle\left|b_{J}\right|^{2}\right\rangle G_{m n}(J) G_{k l}(J),
$$

where (see Appendix)

$$
\begin{aligned}
& \left\langle\left|a_{J}\right|^{2}\right\rangle=\frac{1}{M_{J}} \frac{1}{J^{2}+j_{*}^{2}} ; \quad j_{*}=3 ; \quad M_{J}=\sum_{J=1}^{\infty} \frac{1}{\left(J^{2}+j_{*}^{2}\right)}, \\
& \left\langle\left|b_{J}\left(k_{x}\right)\right|^{2}\right\rangle=\frac{8}{\pi^{2}}\left[\frac{k_{J}}{k_{x}^{2}+k_{J}^{2}}+\frac{1}{2} \frac{k_{J}^{2}}{\left(k_{x}^{2}+k_{J}^{2}\right)^{3 / 2}}\right. \\
& \left.\times \log \left(\frac{\left(k_{x}^{2}+k_{J}^{2}\right)^{1 / 2}+k_{J}}{\left(k_{x}^{2}+k_{J}^{2}\right)^{1 / 2}-k_{J}}\right)\right] \\
& \approx \frac{8}{\pi^{2}} \frac{k_{J}}{k_{J}^{2}+0.5 k_{x}^{2}}, \\
& G_{m n}(J)=\kappa_{0} \zeta_{0} \frac{\mu_{c} N_{0}^{1 / 2}}{g} \int_{0}^{H} d z N(z)^{3 / 2} \\
& \times \sin [\pi J \xi(z)] \varphi_{m}(z) \varphi_{n}(z) .
\end{aligned}
$$

The Lorenzian approximation suggested in Eq. (14) has been used in the past. $^{7,8}$ The approximation gives very good agreement with the precise equation [Eq. (14), left part] and essentially simplifies the form of final equations. Note that for spectral density approximation Eq. (14), the following statement [Eq. (16)] is correct,

$$
\left\langle\rho_{m n}(x) \rho_{k l}(x-\xi)\right\rangle=\left\langle\rho_{m n}(x-\xi) \rho_{k l}(x)\right\rangle .
$$

\section{COMPUTATIONAL ALGORITHM FOR ENTROPY EVOLUTION}

The resultant algorithm ready for computational implementation has the following form:

$$
\frac{\partial\langle\gamma\rangle}{\partial x}=-X\langle\gamma\rangle-\langle\gamma\rangle X^{* T}+Y_{1}(\langle\gamma\rangle)+Y_{2}(\langle\gamma\rangle),
$$

where

$$
\begin{aligned}
X & =\int_{0}^{\infty}\langle M(x) M(x-\xi)\rangle d \xi \\
& =\sum_{n=1}^{N_{a}} \int_{0}^{\infty} e^{-i k_{i} x}\left\langle\rho_{m n}(x) \rho_{n k}(x-\xi)\right\rangle e^{i\left(k_{n}-k_{k}\right) \xi} e^{i k_{k} x} d \xi \\
& =\sum_{J=1}^{N_{J}} \frac{8 \sqrt{2}}{\pi} \frac{1}{M_{J}} \frac{1}{J^{2}+j_{*}^{2}} E\{A(J) B(J)\} E,
\end{aligned}
$$

where the terms are defined by

$$
\begin{aligned}
A(J)= & \left\{A_{m n}(J)\right\}=\left\{\kappa_{0} \zeta_{0} \frac{\mu_{c} N_{0}^{1 / 2}}{g} \int_{0}^{H} N(z)^{3 / 2}\right. \\
& \left.\times \sin [\pi J \xi(z)] \varphi_{n}(z) \varphi_{m}(z) d z\right\}
\end{aligned}
$$

and

$$
\begin{aligned}
B(J)= & \left\{B_{m n}(J)\right\}=\left\{\kappa_{0} \zeta_{0} \frac{\mu_{c} N_{0}^{1 / 2}}{g} \int_{0}^{H} N(z)^{3 / 2}\right. \\
& \left.\times \frac{\sin [\pi J \xi(z)] \varphi_{n}(z) \varphi_{m}(z)}{\left(\sqrt{2} k_{J}-i\left(k_{m}-k_{n}\right)\right)} d z\right\} .
\end{aligned}
$$

The diagonal matrix $E$ is defined as

$$
E=\left\{e^{i k_{n n} x}\right\}
$$

The third and fourth terms are very similar to the first two,

$$
\begin{aligned}
Y_{1}(\langle\gamma\rangle)= & \int_{0}^{\infty}\left\langle M(x-\xi)\langle\gamma\rangle(x) M^{*}(x)^{T}\right\rangle d \xi \\
= & \int_{0}^{\infty}\langle M(x-\xi)\langle\gamma\rangle(x) M(x)\rangle d \xi \\
= & \sum_{n=1}^{N_{a}} \sum_{k=1}^{N_{a}} e^{-i\left(k_{m}-k_{n}\right) x} \int_{0}^{\infty}\left\langle\rho_{m n}(x-\xi) \rho_{k l}(x)\right\rangle \\
& \times e^{i\left(k_{m}-k_{n}\right) \xi} d \xi\left\langle\gamma_{n k}\right\rangle e^{-i\left(k_{k}-k_{l}\right) x} \\
= & \sum_{J=1}^{N_{J}} \frac{8 \sqrt{2}}{\pi} \frac{1}{M_{J}} \frac{1}{J^{2}+j_{*}^{2}}\{E B(J) E\}\langle\gamma\rangle\{E A(J) E\}, \\
Y_{2}(\langle\gamma\rangle)= & \int_{0}^{\infty}\left\langle M(x)\langle\gamma\rangle(x) M^{* T}(x-\xi)\right\rangle d \xi \\
= & \int_{0}^{\infty}\langle M(x)\langle\gamma\rangle(x) M(x-\xi)\rangle d \xi \\
= & \sum_{J=1}^{N_{J}} \frac{8 \sqrt{2}}{\pi} \frac{1}{M_{J}} \frac{1}{J^{2}+j_{*}^{2}}\{E A(J) E\}\langle\gamma\rangle\{E B(J) E\} .
\end{aligned}
$$

The knowledge of mode amplitude correlation $\langle\gamma\rangle$ $=\left\langle U U^{* T}\right\rangle$ will provide an estimate of a variety of acoustic field parameters such as spatial correlations, mean intensity as function of range and depth, and entropy as function of range. For example, the equation for a spatial distribution of mean intensity of random acoustic fields with correlated modes is written

$$
\left\langle|p(x, z)|^{2}\right\rangle=\sum_{m}^{N} \sum_{n}^{N}\left\langle\gamma_{m n}\right\rangle \exp \left(i\left(k_{m}-k_{n}\right) r\right) \varphi_{m}(z) \varphi_{n}(z) .
$$

The estimation of entropy, when random process is close to Gaussian, can be calculated in a similar way.

Everything in the universe eventually moves from order to disorder, and entropy is the measurement of that change. 
Entropy is measure of randomness or the quality of having no apparent order. The word entropy (root from the Greek "entropia") describes the measurement of disorder, chaos, or uncertainty in a system. Specifically, entropy is a logarithmic measure of the number of states with significant probability of being occupied. In information theory, ${ }^{14,15}$ (communications), it is a numerical measure of the uncertainty of an outcome, or measure of information that is missing before reception. ${ }^{14,15}$ It shows the contribution of the scattering by internal waves to signal fluctuations in a long-range propagation. The entropy can be considered as a measure of chaos or complexity of the random process. The complexity of a random process depends on the number of independent components, which in a Gaussian case can be a number of essential non-correlated components in Karhunen-Loeve (K-L) expansion. ${ }^{10}$ The complex envelope of the sound-pressure field at each range step can be represented by a stochastic series with $N(r)$ significant contributions that has been called the dimension of the random process. In more specific form, by the definition, entropy can be calculated through negative Shannon information $I(r)$ of a random process $p(r, z)$ or any of its one to one conversion (for example: $p(r, z) \Longleftrightarrow U(r)$ ),

$$
\begin{aligned}
I(x) & =-\int \log _{2} \operatorname{Pr}(p(x, z)) d \operatorname{Pr}(p(x, z)) \\
& =-\int \log _{2} \operatorname{Pr}(U(x)) d \operatorname{Pr}(U(x)) .
\end{aligned}
$$

The correlation matrix of a Gaussian process defines the entropy. The probability density of a vector $U$ $=\left(u_{1}, u_{2}, \ldots, u_{N}\right)$ of $N$ Gaussian complex random values with nonsingular correlation matrix $K_{U}$ has the following form:

$$
\begin{aligned}
& P_{d f}=\frac{\exp \left(-\left(U-M_{U}\right)^{H} K_{U}^{-1}\left(U-M_{U}\right)\right)}{\pi^{N} \operatorname{det}\left(K_{U}\right)} ; \\
& K_{U}=\left\langle\left(U-M_{U}\right)\left(U-M_{U}\right)^{H}\right\rangle
\end{aligned}
$$

where $K_{U}$ is the complex correlation matrix of the complex random vector $U, M_{U}$ is the mean vector, and the superscript $H$ means Hermitian transpose (complex conjugate and matrix transpose). The entropy, $H$, in the case of a scattering, when random process is close to Gaussian, can be defined in a form, ${ }^{10}$

$$
\begin{aligned}
H(x) & =-I(x)=N \log _{2}(e)+\log _{2}\left(\pi^{N} \operatorname{det}\left(\left\langle\gamma_{m n}\right\rangle\right)\right. \\
& =N \log _{2}(e \pi)+\sum_{j=1}^{N} \log _{2} \lambda_{j}(x)
\end{aligned}
$$

where $\lambda_{j}(x)$ are the eigenvalues of correlation matrix $\left\langle\gamma_{m n}\right\rangle$; $N$ is the number of acoustical modes as in Eq. (2).

For an independent and equal partition of mode energy, $\lambda_{j}(x)=\lambda$ (modal spectrum "white") the entropy is maximum, but for any other spectrum, the entropy is less than this maximum value. Note that this simple definition of entropy is based on the assumption that the Gaussian random process is nonsingular; otherwise, a more rigorous definition must be applied in terms of absolute continuity of probability measures and the Radon-Nikodim derivative. ${ }^{16}$ If the modal intensity is approximately uniform for all significant modes, the entropy will be approximately proportional to the modal spectrum bandwidth. That spectrum will expand with the range and its bandwidth will increase. That is why the entropy rate is related to the divergence or defocusing of very narrow acoustical beam.

In a broadband case, which is more practical case for underwater communication, the same derivation can be provided for a random spectrum of sound pressure $P(f, x, z)$, or a corresponding vector with mode amplitudes $U_{\text {spec }}(f, x)$,

$$
\begin{aligned}
U_{\text {spec }}(f, x) & =\int_{-\infty}^{\infty} U(x, t) \exp (i k 2 \pi f t) d t \\
& =\left\{A_{n}(f) \exp \left(-i k_{n} x\right)\right\}
\end{aligned}
$$

where $\Omega=1 / T$. Note that for the stationary Gaussian process $U(t, x)$, the spectrum harmonics $U_{\text {spec }}(f, x)$ are uncorrelated and independent. The joint entropy of a set of variables is less than or equal to the sum of the individual entropies of the variables in the set (sub-additive). This inequality will become an equality if and only if the variables are statistically independent. The entropy rate does not depend strongly on frequency, and it can be assumed that in a narrow communications frequency range the entropy of all independent frequency components are the same as for the central frequency. As result, the joint entropy rate for a broadband sound waveform will be a product of the entropy rate at the central frequency to the signal base $\triangle F T$,

$$
R_{o e}=(\Delta F T)^{*} R_{e}\left(f_{0}\right),
$$

where $\Delta F$ is the frequency bandwidth, $T$ is the duration of the signal, $\triangle F T$ is the complexity of the signal, $f_{0}$ is the central frequency, $R_{e}\left(f_{0}\right)$ is the entropy rate bit $/ \mathrm{km}$ for the central frequency of the bandwidth, and $R_{o e}$ is the joint entropy.

\section{COMPUTATIONAL EXAMPLE}

A weakly divergent beam (WDB) scattered by a random internal-wave field can be considered as an analog to a Dirac function in channel response estimation theory. The concentrated narrow-angle beam is a simple subject for simulation of acoustic energy scattering. The existence of weakly divergent bundles of rays was discovered and described by Brekhovskikh, ${ }^{12}$ Goncharov, ${ }^{17}$ and Petukhov. ${ }^{18}$ The rigorous necessary condition for the formation of a strictly nondivergent beam in a full wave approach is equivalent to the local linearity of the mode wave number as a function of mode order. ${ }^{10,11}$ An underwater wave-guide with a canonical Munk sound speed profile does not exactly satisfy that condition, but beam divergence is very weak. The mode shapes for the canonical Munk sound speed profile are shown in the Fig. 1. A beam generated in the Munk sound waveguide, from the depth $2000 \mathrm{~m}$ at the frequency $250 \mathrm{~Hz}$, calculated by a normal mode expansion after scattering by internal waves is 


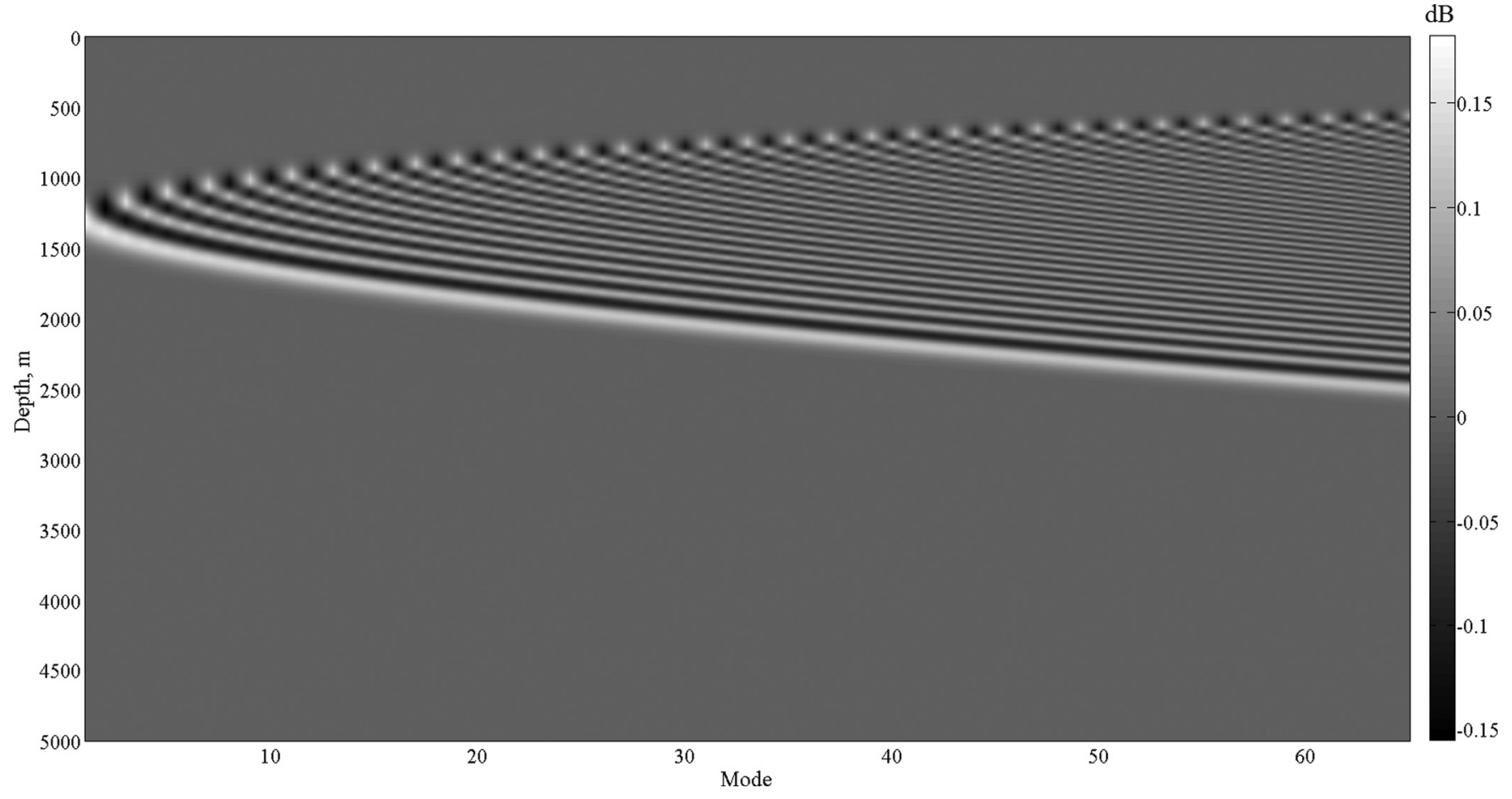

FIG. 1. Mode shapes for the canonical Munk sound speed profile.

shown in the Fig. 2. The vertical dimension of the Gaussian starting aperture is roughly $150 \mathrm{~m}$ (25 wavelengths),

$$
p=\sqrt{\kappa_{0} / \Delta} \exp \left\{-\left(\kappa_{0} / \Delta\right)^{2}\left(z-z_{s}\right)^{2}\right\}
$$

where $\kappa_{0}=2 \pi f / c_{0}, c_{0}=1500 \mathrm{~m} / \mathrm{s}, z_{s}$ is the sound source depth, and the width factor $\Delta$ was adjusted to value 60 to achieve narrow beams. Here and in the subsequent analysis all cylindrical spreading factors were removed.

\section{A. Internal waves model parameters}

The random internal wave model is based on a twodimensional approximation of the Garrett-Munk spectrum in

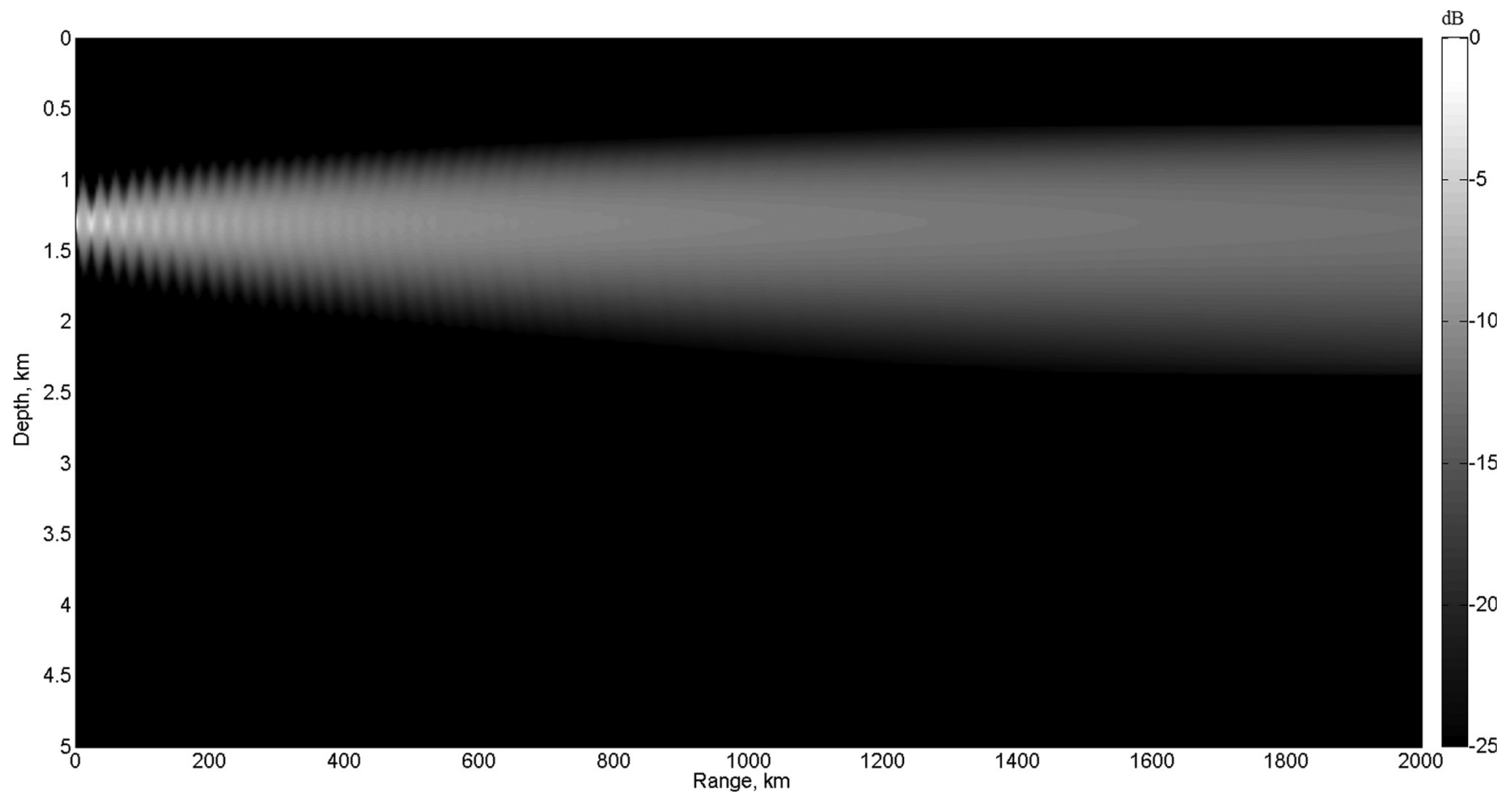

FIG. 2. Intensity of scattered beam from the depth $1300 \mathrm{~m}$, depth of underwater acoustic channel. 


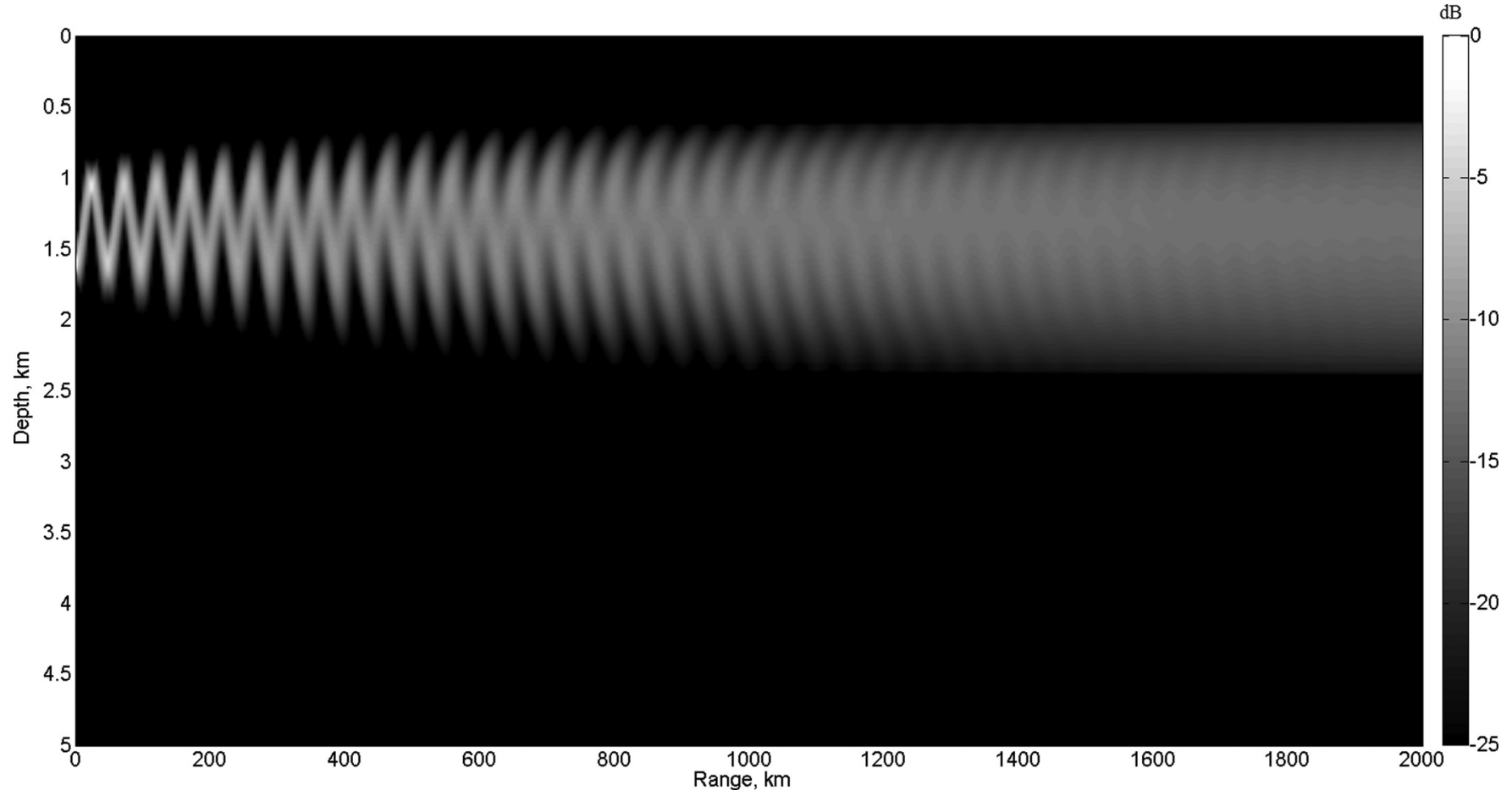

FIG. 3. Intensity of scattered beam from the depth $1600 \mathrm{~m}$.

accordance with Colosi and Brown ${ }^{3}$ with internal wave index: $J=1: N_{J}, N_{J}=50$.

The mean intensity of a narrow beam emitted from the depth of the underwater sound channel $1300 \mathrm{~m}$ at the frequency of $250 \mathrm{~Hz}$ and scattered by random internal wave field is shown in Fig. 2. The mean intensity of a narrow beam emitted from the depth 1600 and $2000 \mathrm{~m}$ and the frequency of $250 \mathrm{~Hz}$ are shown in Figs. 3 and 4, respectively.
The calculation used formula (28) after the differential Eq. (21) were solved.

The complexity of the acoustical scattered field directly depends on the number of independent components in its structure or on the number of eigen functions of its correlation function. The number of eigen functions as a function of range for the three considered cases is shown in Fig. 5. In that example, the frequency of the acoustic signal was

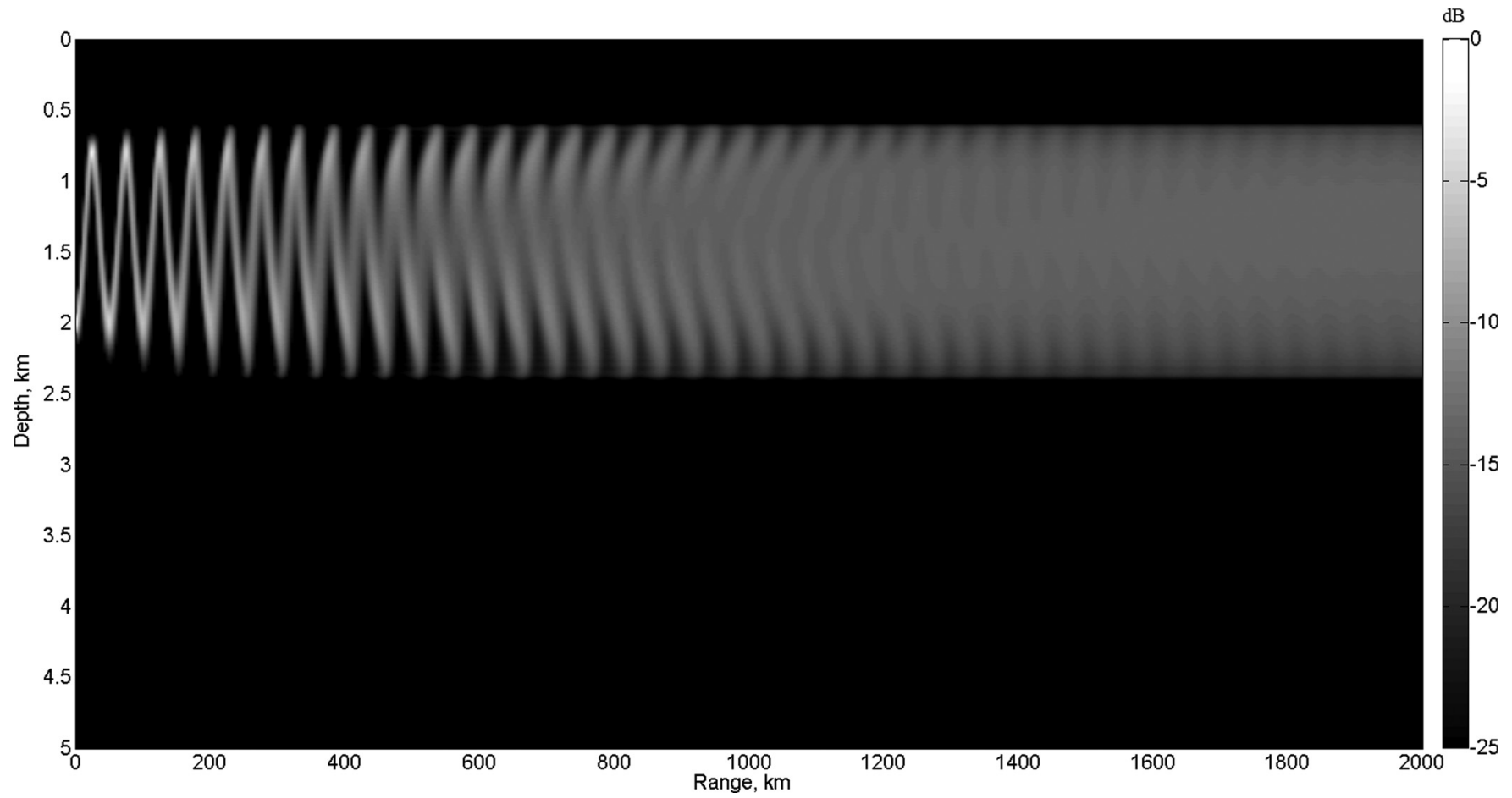

FIG. 4. Intensity of scattered beam from the depth $2000 \mathrm{~m}$. 


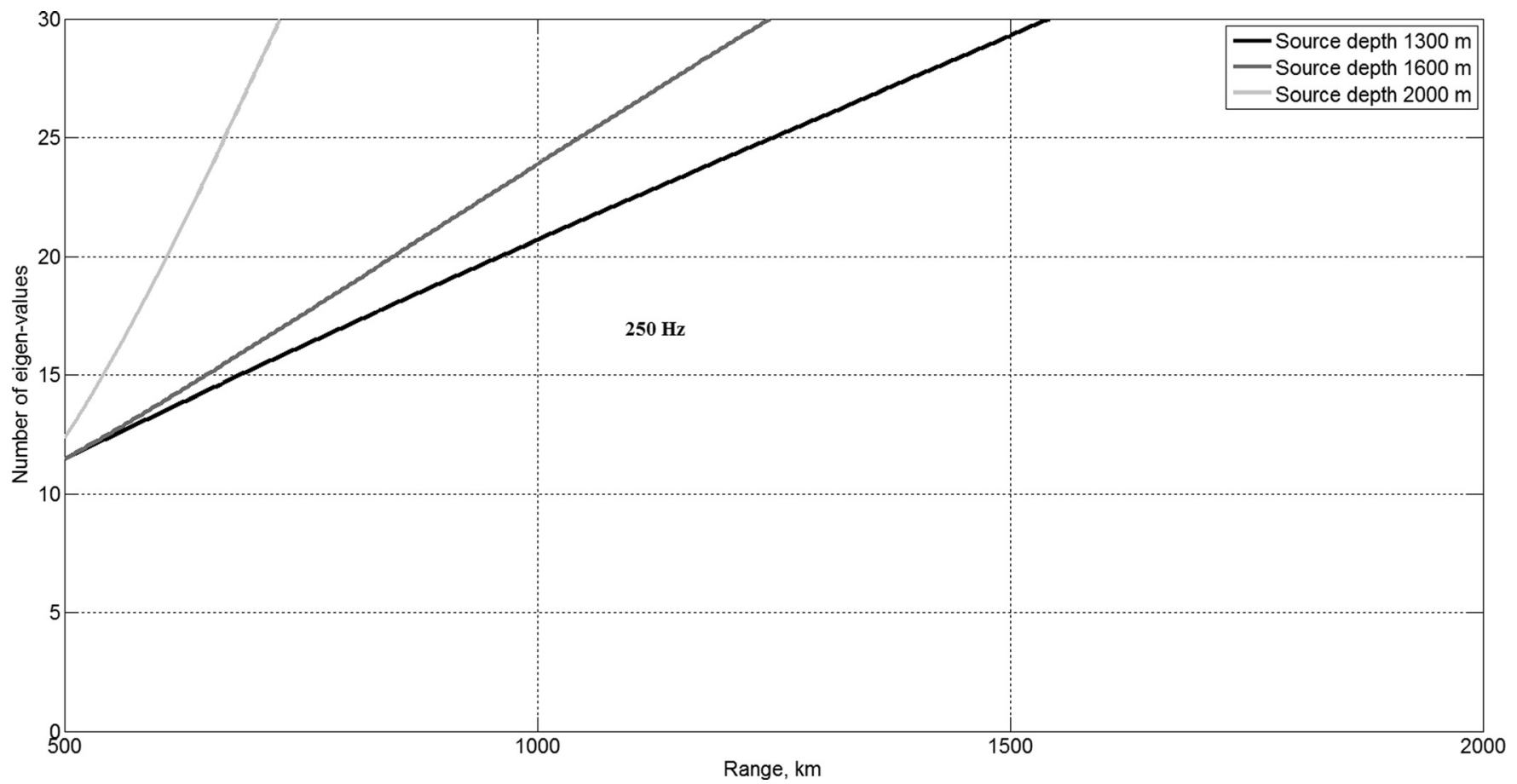

FIG. 5. Eigen-function number evolution.

$250 \mathrm{~Hz}$ and the sound source depth was varied among 1300 , 1600 , and $2000 \mathrm{~m}$. The eigenvalues were considered significant if they were $75 \%$ of the maximum value or greater. It can be noticed that for acoustical beams close to the channel axis $(1300 \mathrm{~m})$, the number of significant eigenvalues grows linearly with the range.

Entropy levels calculated directly by formula (31) for different frequencies and different sound source depths are presented in the Figs. 6 and 7. It can be noticed that for all considered examples after $1500 \mathrm{~km}$ range, the entropy is increasing with a slowly declining rate. The physical meaning of entropy rate is the information (coherency) loss during propagation in the random ocean at the range $1 \mathrm{~km}$. Those entropy rates for different frequencies and different depths of sound source are presented in the Figs. 8 and 9. The entropy rates as a function of range at the frequency $250 \mathrm{~Hz}$ for three different of sound source depths, 1300, 1600, and $2000 \mathrm{~m}$, are shown in Fig. 10. It is interesting that although entropies are monotonically growing, the rate at the beginning of the process is not stable. The rate is oscillating

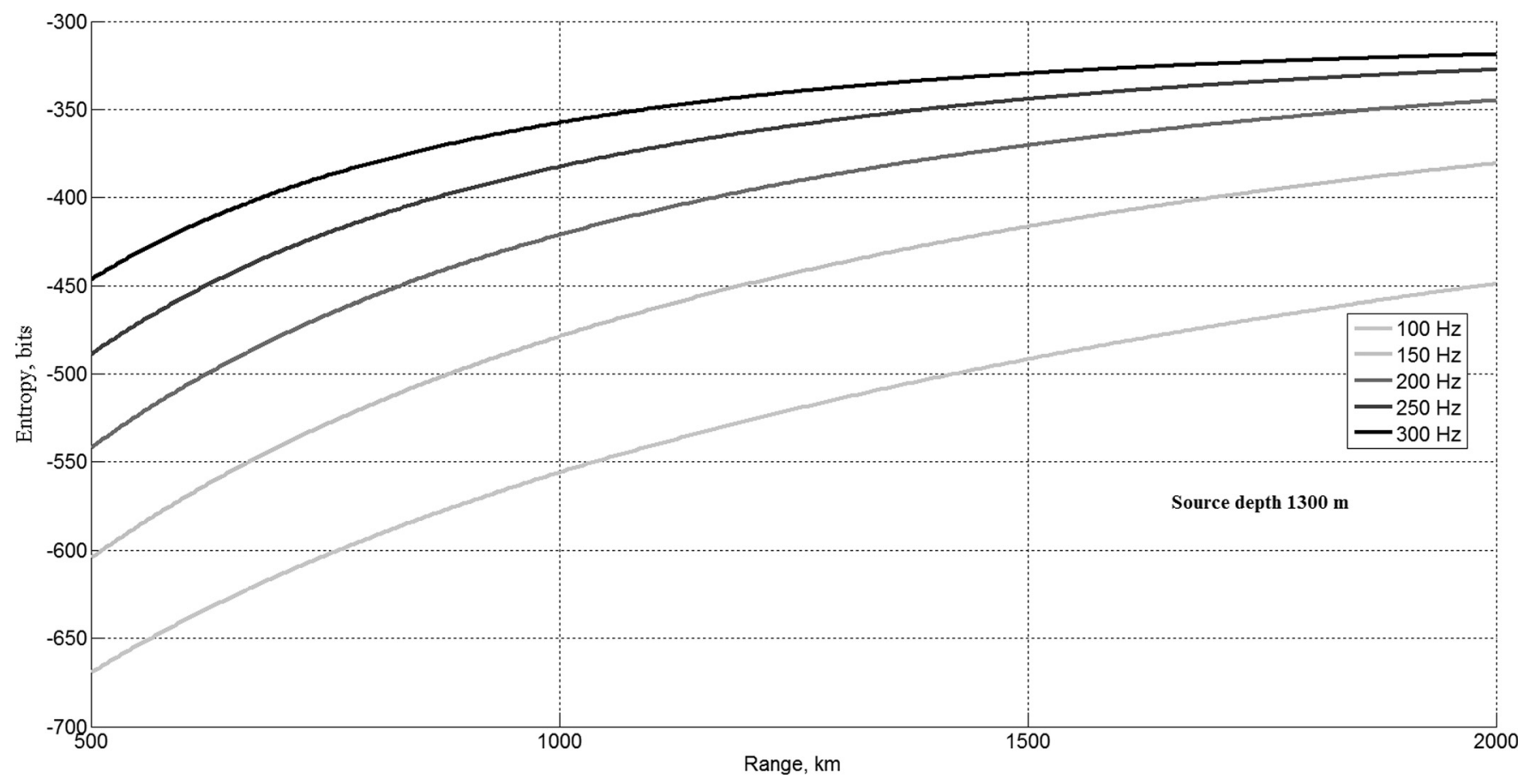

FIG. 6. Entropy evolution over range $2000 \mathrm{~km}$, sound source at the depth of underwater acoustic channel-1300 m. 


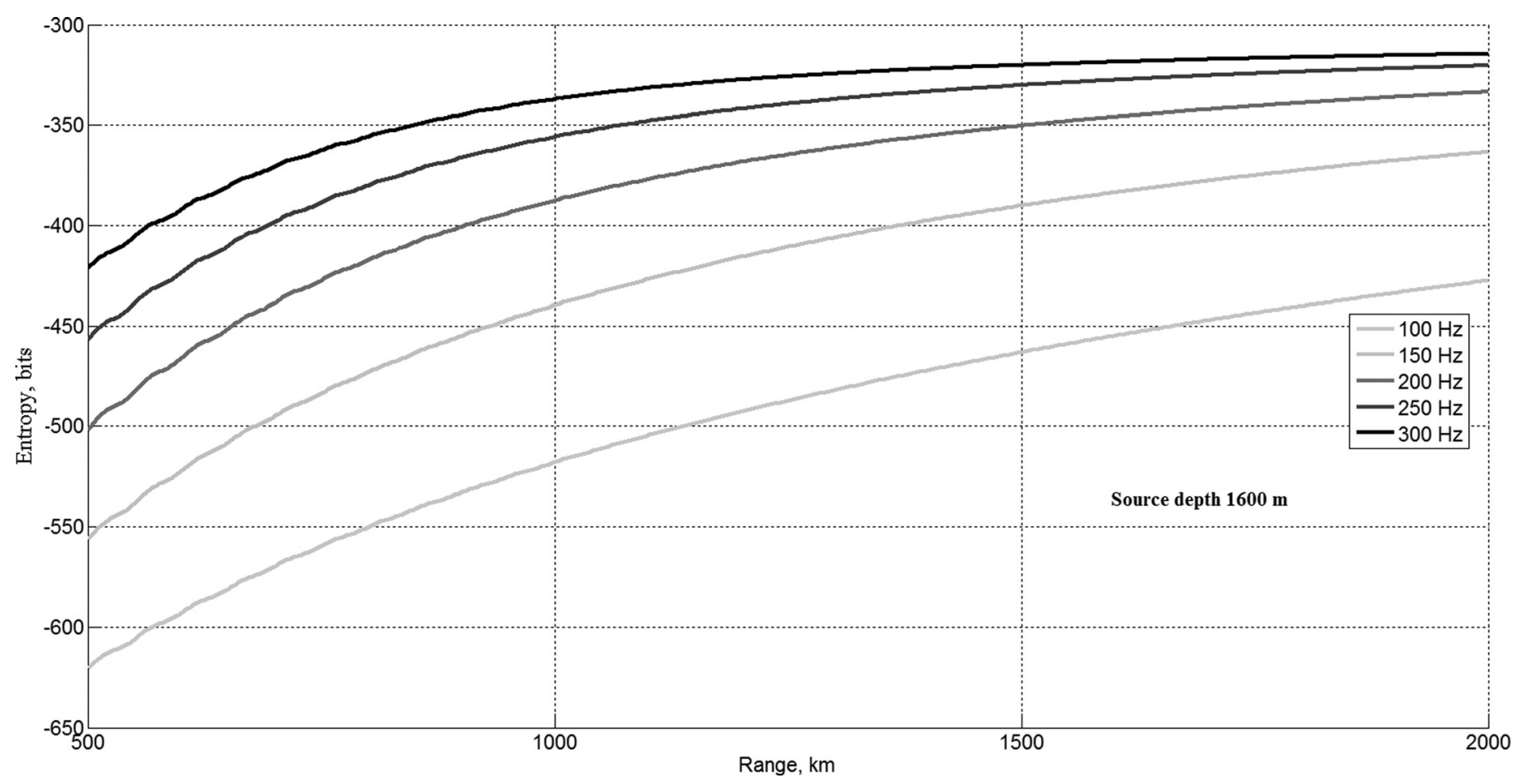

FIG. 7. Entropy evolution over range $2000 \mathrm{~km}$, sound source depth—1600 m.

because of the diffraction of the narrow beams. At that part of the curve, the process cannot be considered as entirely chaotic or random; what is seen is ordinary wave diffraction. At the end of the range, the entropy rate is stabilized to some constant value, depending from beam angle. These rates are the same order of magnitude as the Lyapunov exponents for ray chaos. Specifically, at the axis of the underwater channel $(1300 \mathrm{~m})$, entropy rate at the distance $2000 \mathrm{~km}$ is decreasing with the frequency from the level of 0.7 at $100 \mathrm{~Hz}$ to 0.2 at $300 \mathrm{~Hz}$. For the larger propagation angle beams with a
$1600 \mathrm{~m}$ source depth (or larger), the entropy rate is decreasing from value 0.06 at $100 \mathrm{~Hz}$ to the value 0.01 at $300 \mathrm{~Hz}$. These asymptotical values will be compared with K-S entropy, which is a specific measure of ray chaos instability.

\section{RAY CHAOS AND K-S ENTROPY}

There is a strong connection between wave and ray dynamics in underwater sound scattering. Shannon entropy in a coupled mode approach is directly related to the acoustical

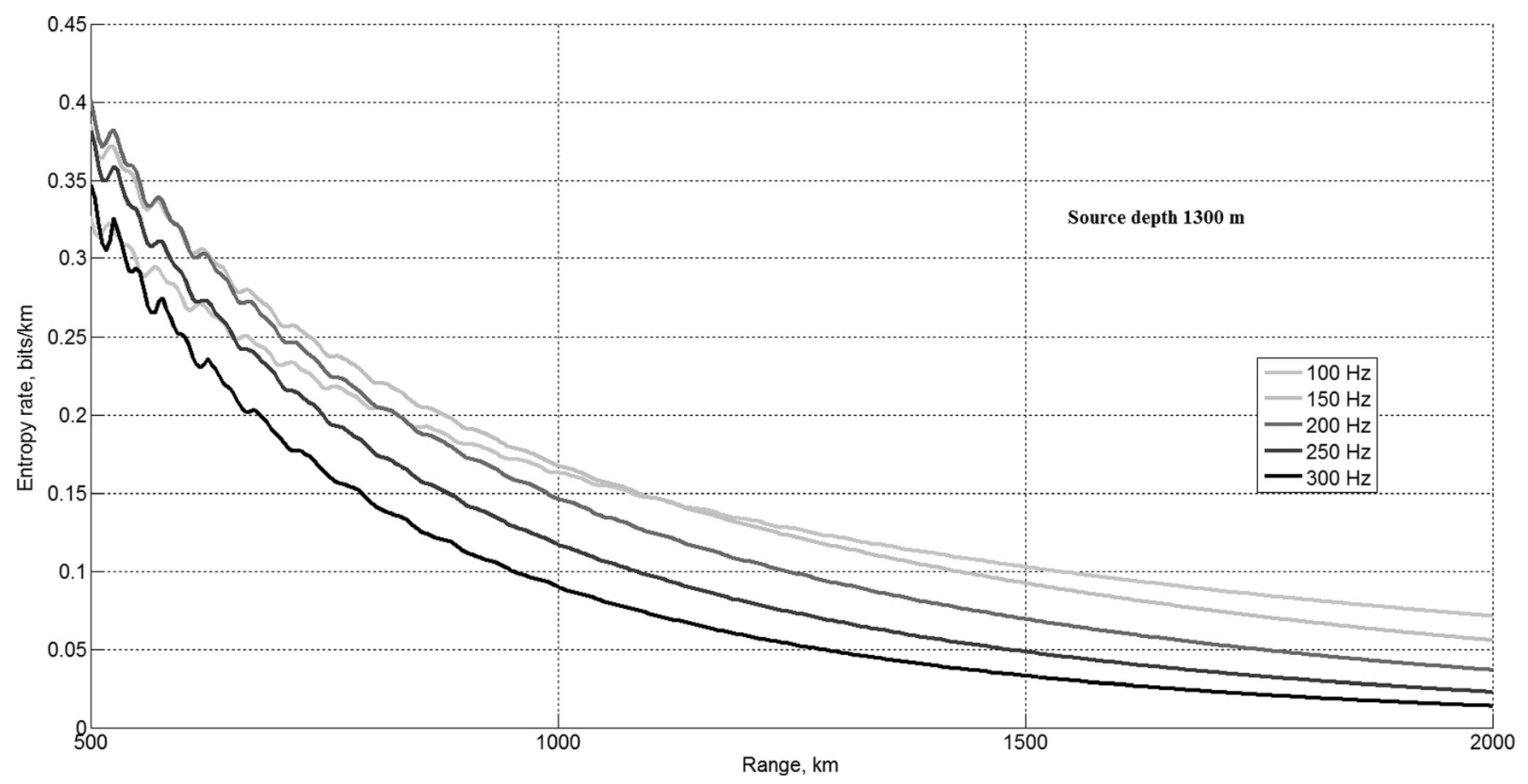

FIG. 8. Entropy rate as function of range, sound source depth $1300-\mathrm{m}$. 


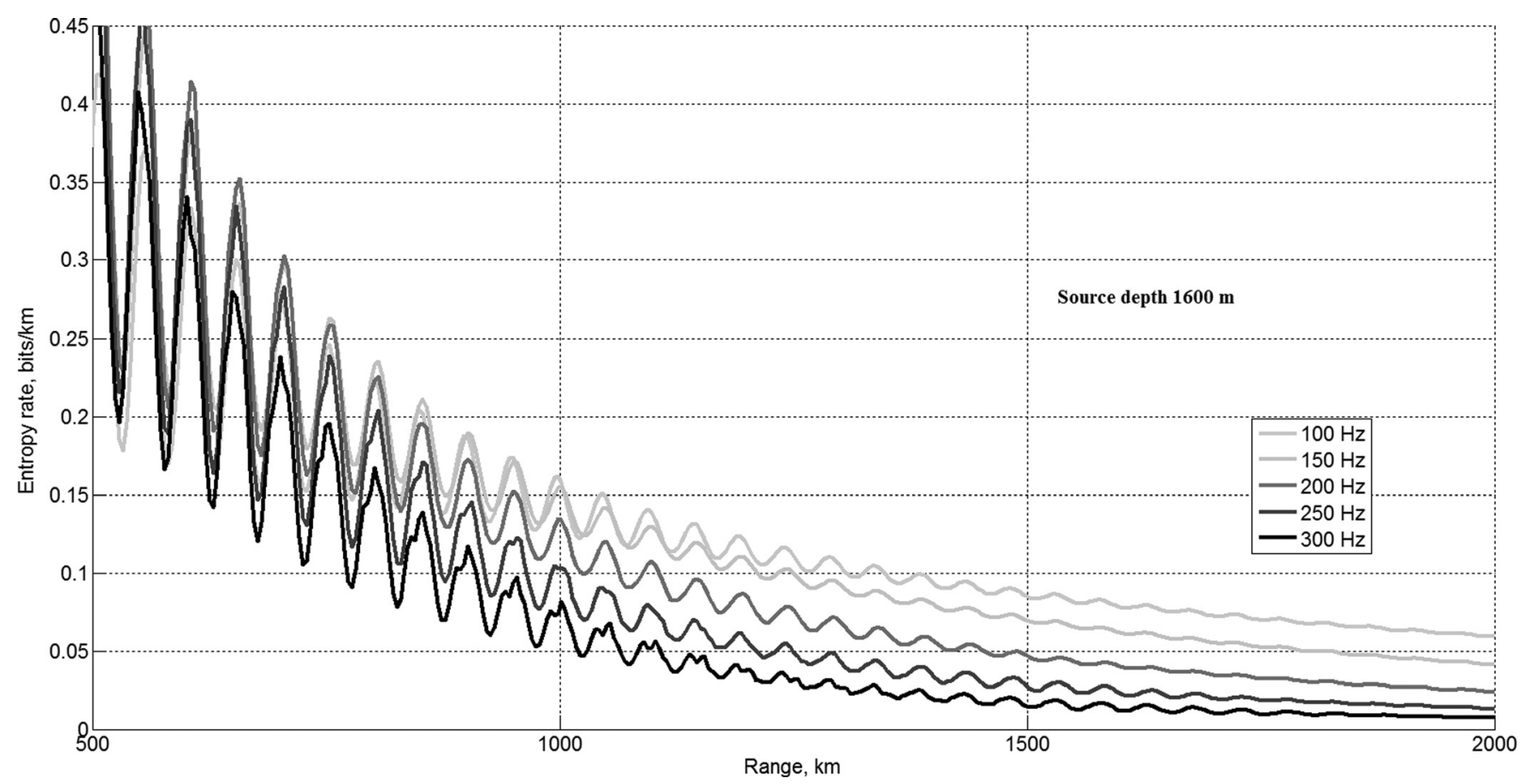

FIG. 9. Entropy rate as function of range, sound source depth $1600-\mathrm{m}$.

ray stability and ray chaos theory. Ray trajectories in ocean acoustic propagation through internal waves are chaotic or unstable to perturbations in the medium and in the initial conditions. $^{1,2}$ The chaotic nature of ray propagation in the ocean is quantified using the Lyapunov exponent $\nu_{L}$, which is derived from a stability analysis of the ray path. ${ }^{1}$ The instability can be expressed in terms of information theory ${ }^{19,20}$ by K-S entropy $h_{K S}$,

$\lim _{|s| \rightarrow \infty} I(s) / s=h_{K S}$, where information $I(s)$ is associated with a segment of ray trajectory of length $s$. The K-S entropy can be considered to be entropy per unit length of propagation trajectory. For bounded dynamical systems like underwater sound $\nu_{L}=h_{K S},{ }^{21}$ a consequence of the chaotic nature of ray paths is that the number of eigen-rays connecting a source and a receiver will grow exponentially with range, leading to an exponential increase in wave field complexity. ${ }^{22}$ Thus the exponential increase in wave field complexity is examined using the Lyapunov exponent (a measure of the K-S

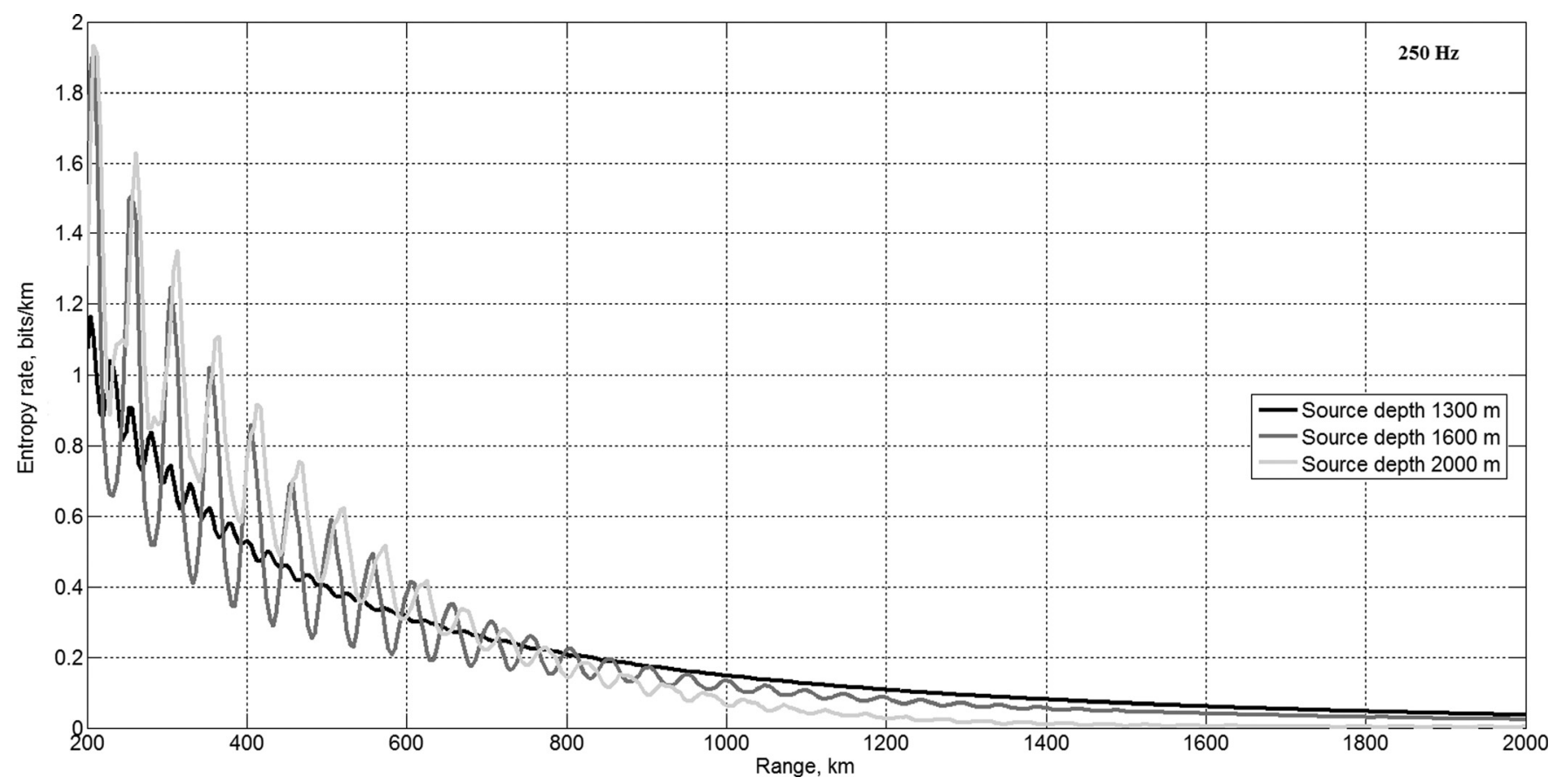

FIG. 10. Entropy rate as function of range, frequency-250 Hz. 
entropy), which is to be compared to the computed rate of Shannon entropy. The Lyapunov exponent is derived from the stability equations, and is given by

$$
\nu_{L}=\lim _{x \rightarrow \infty} \log |\operatorname{Tr}(J)| / x
$$

where $\operatorname{Tr}(J)$ is the trace or sum over the diagonal elements of the stability matrix $J{ }^{1,23}$ A finite range stability exponent can be estimated by $\nu=\log |\operatorname{Tr}(J)| / x$. This finite range stability exponent has the property ${ }^{23}$ that $\nu_{L}=\langle\nu\rangle \sim h_{K S}$, where the expectation values imply averaging over realizations of the ocean internal-wave field. The Lyapunov exponent was calculated numerically ${ }^{10}$ for the ray initial condition $z(0)=1300 \mathrm{~m}, p(0)=0$. An explicit adaptive Runge-Kutta algorithm was used to calculate 200 realization of ray trajectories with the preceding initial conditions. The calculation yields an estimate $h_{K S}$ of $0.02 \mathrm{bit} / \mathrm{km}$ for the axial ray, decreasing linearly to $0.012 \mathrm{bit} / \mathrm{km}$ for the $2000-\mathrm{m}$ source depth ray. These values are comparable to other calculations of the Lyapunov exponent for ray propagation through ocean internal waves. ${ }^{1,2}$ The value of $0.02 \mathrm{bit} / \mathrm{km}$ for the entropy rate $h_{K S}$ is exactly equal to the long range value of the Shannon entropy rate for the $300 \mathrm{~Hz}$ axial beam case. For the source depths of 1600 and $2000 \mathrm{~m}$, the long range entropy rate at $300 \mathrm{~Hz}$ is $0.01 \mathrm{bit} / \mathrm{km}$. For the deeper source depths, the entropy rate is smaller because a limited number of acoustical modes were used in the simulation. Comparison of the physical wave chaos entropy rate to the $\mathrm{K}-\mathrm{S}$ entropy under the same propagation conditions shows that the levels are converging at long range and high frequency. This condition occurs because diffraction effects become less and less important. However, in comparing these entropy values to the computed gradients of Shannon entropy for the complex envelope, it must be noted that the complex envelope includes information about both phase and amplitude, while $h_{K S}$ is only a measure of the soundfield energy characteristics, such as intensity and angular power density. It must be emphasized here that the comparison between K-S entropy and full-wave Shannon entropy rate is crude as we have only compared the K-S entropy of one ray, while the Shannon entropy involves a bundle of rays with a range (albeit small) of initial conditions. It is known, for example, that there can be significant variability of Lyapunov exponent as a function of initial conditions due to the structure of the background sound-speed profile. ${ }^{1,2}$ So the information gradient of the full-field entropy cannot be exactly equal to the K-S entropy.

The comparison of Shannon or physical entropies curves $H(x)$ of the complex envelope of the full-field simulation with $h_{K S}$ shows that the evolution of narrow-beam entropy has two stages (see Figs. 5-10). In the first stage, the beamwidth and spatial spectrum are growing rapidly due to the wave diffraction (Figs. 5-10 for range $x$ smaller than $1500 \mathrm{~km}$ ). The second stage occurs approximately near saturation $(x>1500 \mathrm{~km})$; the entropy gradient slows down and tends to a level that at high frequencies is approximately equal the K-S entropy of the corresponding chaotic ray trajectories. This second stage can be called chaotic from both a full-wave and ray perspective because entropy is monotonically growing with the gradient determined by the averaged Lyapunov exponent. Simultaneously the beam width, the spatial spectrum width, and the dimension of the random process are all approximately linearly increasing. The linear entropy gradient shows that near saturation of fluctuations, ray chaos phenomenon becomes the main factor determining the properties of scattering processes.

\section{CONCLUSION}

The derived transport theory equations for the second order moments of an acoustic field in an ocean with a random internal wave field give rigorous predictions of the entropy rate and informational losses. The equations were used for our entropy range evolution study in an ocean consisting of Garrett-Munk internal waves. At long range, nearsaturation finite-frequency narrow beams show a slowly decreasing entropy rate that does not depend strongly on frequency. This full-wave rate of entropy is of the same order of magnitude as the K-S entropy and finite-range Lyapunov exponents for the ray trajectories associated with this beam. Comparison of Shannon entropy rate and K-S entropy has shown that some differences between them can be explained by the different physical nature of the information carriers. Parameters of wave scattering and ray chaos are well matched. The correspondence between full-wave and ray entropies suggests a correspondence between full-wave scattering and ray chaos near statistical saturation. However, the roots of that correspondence are not obvious and should be considered for further investigation. The analysis shows that in a random internal-wave field with the Garrett-Munk spectrum close to the axis of the canonical Munk underwater wave-guide, the entropy decreases at the rate as $0.01-0.05 \mathrm{bit} / \mathrm{km}$ per one degree of diversity. Such small level of entropy rate during propagation through random internal-wave field shows that scattering by internal waves should not be considered as an essential limitation for data rate and channel capacity.

\section{ACKNOWLEDGMENT}

This work was supported in part by Office of Naval Research grant.

\section{APPENDIX}

The following stochastic differential equations basics have been gathered and briefly presented in the Appendix for the convenience of the reader. The original theory can be found in details in the papers. ${ }^{11}$

Let us consider the system of stochastic differential equations in a form in a matrix form,

$$
\frac{\partial U}{\partial x}=i M U
$$

where $U$ is the stochastic vector process. Particularly, in that paper, it is related to acoustic mode coefficients and random matrix of mode coupling coefficients by equations described in the paper as follows: 


$$
\begin{aligned}
& U=\left\{A_{n} \exp \left(-i k_{n} x\right)\right\}, \quad U(x=0)=U_{0}, \\
& M(x)=\left\{-e^{-i k_{m} x} \rho_{m n}(x) e^{i k_{n} x}\right\},
\end{aligned}
$$

$M$ is a square symmetrical matrix. The formal general solution of Eq. (A1) has a form of a time ordering ("o") exponent,

$$
\begin{aligned}
& U=\exp _{o}\left(i \int_{0}^{x} M\left(x^{\prime}\right) d x^{\prime}\right) U_{0}, \\
& \exp _{o}\left(i \int_{0}^{x} M\left(x^{\prime}\right) d x^{\prime}\right) \\
&=\left(\sum_{m=1}^{\infty} i^{m} \int_{0}^{x} \int_{0}^{x_{1}} \ldots \int_{0}^{x_{m-1}} M\left(x_{1}\right) \ldots M\left(x_{m-1}\right) d x_{1} \ldots d x_{m-1}\right)_{o} .
\end{aligned}
$$

It is important to note that (A3) is a formal solution, which can be verified by substitution and being very careful about collecting terms of the sum in the time ordering, see the Ref. 11.

If a random process $M(x)$ and $\left(\int_{0}^{x} M\left(x^{\prime}\right) d x^{\prime}\right)$ are Gaussian, then all its cumulants ${ }^{13}$ with order more than 2 are equal to zero and cumulant $\left\langle\left\langle M\left(x_{1}\right) M\left(x_{2}\right)\right\rangle\right\rangle=\left\langle M\left(x_{1}\right)\right.$ $\left.M\left(x_{2}\right)\right\rangle$, where $\langle\langle\rangle$.$\rangle is the cumulant operator; \langle$.$\rangle is the aver-$ age operator. The components $\left\langle\left\langle M^{n}\left(x_{i}\right) d x^{n} M\left(x_{j}\right) d x_{j} \ldots\right\rangle\right\rangle$ in a general cumulate exponent form vanish for all orders $n>1$, when $d x->0$, and we obtain the simple cumulant integral in the exponent index. ${ }^{13}$ It allows us to write equation for the averaged exponent,

$$
\begin{aligned}
& \left\langle\exp _{o}\left(i \int_{0}^{x} M\left(x^{\prime}\right) d x^{\prime}\right)\right\rangle \\
& =\exp _{o}\left(\sum _ { m = 1 } ^ { \infty } i ^ { m } \int _ { 0 } ^ { x } d x _ { 1 } \ldots \int _ { 0 } ^ { x _ { m } } d x _ { m } \left\langle\left\langleM\left(x_{1}\right)\right.\right.\right. \\
& \left.\left.\left.\quad \times M\left(x_{2}\right) \ldots M\left(x_{m}\right)\right\rangle\right\rangle d x_{1} \ldots d x_{m}\right) \\
& =\exp _{o}\left(-\int_{0}^{x} d x_{1} \int_{0}^{x_{1}} d x_{2}\left\langle M\left(x_{1}\right) M\left(x_{2}\right)\right\rangle\right) .
\end{aligned}
$$

After taking derivative from Eq. (A5), in a lowest order approximation assuming, that M-operators are commuting, and we can disregard range ordering. In that form, the equations are approximation of a precise solution. The Moperators commute condition for example (sufficient but not necessary), when process is delta-correlated or in a case of small scattering on one step propagation process but not small on a large scale.

$$
\frac{d}{d x}\langle U\rangle=-\int_{0}^{x}\langle M(x) M(x-\xi)\rangle d \xi\langle U\rangle .
$$

Numerical estimations show that with a very small error at the beginning of process, we can replace the integral on range independent equal to the value with infinity limits

$$
\frac{d}{d x}\langle U\rangle=-\int_{0}^{\infty}\langle M(x) M(x-\xi)\rangle d \xi\langle U\rangle .
$$

The second order moment equation follows from the equation for random variable $\gamma=U U^{* T}$, where * is the conjugate operator and $T$ is the transposition. Let us return back to Eq. (A1) and multiply it from the right side by matrix $U^{* T}$, then conjugate Eq. (A1) and multiply it from the left side by $U$. Summing up these two result equations gives the differential equation for matrix $U U^{* T}$ [Eq. (A8)],

$$
\frac{\partial U U^{* T}}{\partial x}=i\left(M U U^{* T}-U U^{* T} M^{* T}\right) ; \quad \frac{\partial \gamma}{\partial x}=i\left(M \gamma-\gamma M^{* T}\right) .
$$

The mean evolution equation corresponding to the matrix Eq. (17) can be obtained by transforming equation into the form equivalent to Eq. (A1). The transformation is based on reshaping $N \times N$ matrix $\gamma=\left\{\gamma_{m n}\right\}=U U^{* T}$ into $N^{2} \times 1$ vector-column $\tilde{\gamma}=\left\{\gamma_{i}\right\}$ formed by consecutively stacking the columns of $\gamma$ in accordance with rule: $i=m+(n-1)$ $\times N$. The same transformation sets up a correspondence between a square matrix $\left(M \gamma-\gamma M^{* T}\right)$ and vector-column $\left(I \otimes M-M^{*} \otimes I\right) \tilde{\gamma}$ and finally between Eq. (A13) and its tensor form Eq. (A14),

$$
\frac{\partial \tilde{\gamma}}{\partial x}=\tilde{M} \tilde{\gamma}
$$

where $\tilde{M}=\left(I \otimes M-M^{*} \otimes I\right) ; \otimes$ is the Kronecker tensor product with the following properties:

$$
\begin{aligned}
& C=A \otimes B: \quad c_{p q}=\left\{a_{k l} B\right\}=\left\{a_{k l} b_{m n}\right\} ; \\
& p=m+(k-1) N ; \quad q=n+(l-1) N, \\
& (A \otimes B)(C \otimes D)=(A C \otimes B D), \\
& (A \otimes B) \tilde{\gamma} \rightarrow B \gamma \gamma^{T},
\end{aligned}
$$

where $A$ and $B$ are arbitrary $N \times N$ matrixes and $N^{2} \times 1$ vector-column $\tilde{\gamma}$ related with $\gamma$ by inverse transformation of dimensions $N^{2} \times 1 \rightarrow N \times N$, shown here and in Eq. (A12) by arrow " $\rightarrow$." The correlation evolution equation can be readily obtained in a form,

$$
\frac{d\langle\tilde{\gamma}\rangle}{d x}=-\int_{0}^{\infty}\langle\tilde{M}(x) \tilde{M}(x-\xi)\rangle d \xi\langle\tilde{\gamma}\rangle .
$$

Next transformation is based on the abovementioned property [Eq. (A11)] of tensor product

$$
\begin{aligned}
\frac{\partial\langle\tilde{\gamma}\rangle}{\partial x}= & {\left[-I \otimes\left[\int_{0}^{\infty}\langle(M(x) M(x-\xi)\rangle d \xi]\right.\right.} \\
& -\left[\int_{0}^{\infty}\left\langle\left(M^{*}(x) M^{*}(x-\xi)\right\rangle d \xi\right] \otimes I\right. \\
& +\int_{0}^{\infty}\left\langle M^{*}(x) \otimes M(x-\xi)\right\rangle d \xi \\
& \left.+\int_{0}^{\infty}\left\langle M^{*}(x-\xi) \otimes M(x)\right\rangle d \xi\right]\langle\tilde{\gamma}\rangle .
\end{aligned}
$$


Moreover, we can avoid a large dimension $N^{2} \times N^{2}$ matrix $\tilde{M}$ and return back to matrix $M$ (with initial dimension $N \times N)$ and equation for correlation matrix $\langle\gamma\rangle$, if we apply Eq. (A12), ${ }^{10}$

$$
\begin{aligned}
\frac{\partial\langle\gamma\rangle}{\partial x}= & -\int_{0}^{\infty}\langle M(x) M(x-\xi)\rangle d \xi\langle\gamma\rangle \\
& -\langle\gamma\rangle \int_{0}^{\infty}\left\langle M^{*}(x) M^{*}(x-\xi)\right\rangle^{T} d \xi \\
& +\int_{0}^{\infty}\left\langle M(x-\xi)\langle\gamma\rangle(x) M^{* T}(x)\right\rangle d \xi \\
& +\int_{0}^{\infty}\left\langle M(x)\langle\gamma\rangle(x) M^{* T}(x-\xi)\right\rangle d \xi .
\end{aligned}
$$

The matrix $M(x)$ is determined by internal-waves model $\left(\right.$ Colosi and Brown $\left.{ }^{3}\right)$, as it follows.

The internal-waves random field $\zeta(x, z)$ mode series presentation has a form:

$$
\begin{aligned}
& \zeta(x, z)=\zeta_{0}\left(\frac{N_{0}}{N(z)}\right)^{1 / 2} \sum_{J=1}^{\infty} a_{J} \sin [\pi J \xi(z)] \int_{-\infty}^{\infty} d k_{x} b_{J}\left(k_{x}\right) e^{i k_{x} x}, \\
& \zeta\left(k_{x}, z\right)=\zeta_{0}\left(\frac{N_{0}}{N(z)}\right)^{1 / 2} \sum_{J=1}^{\infty} a_{J} \sin [\pi J \xi(z)] b_{J}\left(k_{x}\right),
\end{aligned}
$$

where $\xi(z)=1 / N_{0} B \int_{z}^{D} N\left(z^{\prime}\right) d z^{\prime}$ is WKBJ stretched vertical coordinate, $B=N_{0} / \int_{0}^{D} N\left(z^{\prime}\right) d z^{\prime}, N(z)$ is buoyancy frequency, $N_{0}=\pi / 600$ is the surface extrapolated buoyancy frequency, ${ }^{3}$ and the internal-wave mode coefficient $a_{J}$ is a complex Gaussian random variable. Using the paper definition Eq. (5), we have

$$
\begin{aligned}
\hat{\rho}_{m n}\left(k_{x}\right)= & \kappa_{0} \zeta_{0} \sum_{J=1}^{\infty} a_{J} b_{J}\left(k_{x}\right) \int_{0}^{D} d z \frac{1}{c_{0}}\left(\frac{\partial c}{\partial z}\right)_{p}(z) \\
& \times\left(\frac{N_{0}}{N(z)}\right)^{1 / 2} \sin [\pi J \xi(z)] \varphi_{m}(z) \varphi_{n}(z) \\
= & \kappa_{0} \zeta_{0} \frac{\mu_{c} N_{0}^{1 / 2}}{g} \sum_{J=1}^{\infty} a_{J} b_{J}\left(k_{x}\right) \int_{0}^{D} d z N(z)^{3 / 2} \\
& \times \sin [\pi J \xi(z)] \varphi_{m}(z) \varphi_{n}(z),
\end{aligned}
$$

where $J$ is index for internal waves, ${ }^{3} J=1: N_{J} ; \mu_{c}=24.5$ (dimensionless); $\varsigma_{0}=7.3 \mathrm{~m} ; g=9.8 \mathrm{~m} / \mathrm{s}^{2}$,

$$
\begin{aligned}
& \rho_{m n}(x)=\int_{-\infty}^{\infty} d k \hat{\rho}_{m n}(k) e^{-i k x} \\
& \hat{\rho}_{m n}(k)=\frac{1}{2 \pi} \int_{-\infty}^{\infty} d x \rho_{m n}(x) e^{i k x} .
\end{aligned}
$$

The paper Eq. (12) for coupled mode correlation matrix $\left\langle\hat{\rho}_{m n}\left(k_{x}\right) \hat{\rho}_{k l}^{*}\left(k_{x}\right)\right\rangle$ follows directly from that simplified internal wave analytic model. That matrix determines statistics of random matrix $M(x)$ and finally the form of the Eq. (17) for correlation matrix.

${ }^{1}$ M. G. Brown, J. A. Colosi, S., Tomsovic, A. L. Virovlyansky, M. A. Wolfson, and G. M. Zaslavsky, "Ray dynamics in a long range deep ocean sound propagation," J. Acoust. Soc. Am. 113(5), 2533-2547 (2003).

${ }^{2}$ M. G. Brown, F. J. Beron-Vera, I. I. Rypina, and I. A. Vdovydchenkov, "Rays, modes, wavefield structure, and wavefield stability," J. Acoust. Soc. Am. 117(3), 1607-1610 (2005).

${ }^{3}$ J. A. Colosi and M. G. Brown, "Efficient numerical simulation of stochastic internal-wave induced sound-speed perturbation fields," J. Acoust. Soc. Am. 103(4), 2232-2235 (1998).

${ }^{4}$ J. A. Colosi and A. K. Morozov, "Statistics of normal mode amplitudes in an ocean with random sound-speed perturbations: Cross-mode coherence and mean intensity," J. Acoust. Soc. Am. 126(3), 1026-1035 (2009).

${ }^{5}$ J. A. Colosi, T. F. Duda, and A. K. Morozov, "Statistics of low-frequency normal-mode amplitudes in an ocean with random sound-speed perturbations: Shallow-water environments," J. Acoust. Soc. Am. 131(2), 1749-1761 (2012).

${ }^{6}$ R. Dashen, S. M. Flatté, and S. A. Reynolds, "Path-integral treatment of acoustic mutual coherence functions for rays in a sound channel," J. Acoust. Soc. Am. 77(5), 1716-1722 (1985).

${ }^{7}$ L. B. Dozier and F. D. Tappert, "Statistics of normal mode amplitudes in a random ocean. I. Theory," J. Acoust. Soc. Am. 63(2), 353-365 (1978).

${ }^{8}$ L. B. Dozier and F. D. Tappert, "Statistics of normal mode amplitudes in a random ocean. II. Computations,” J. Acoust. Soc. Am. 64(2), 533-547 (1978).

${ }^{9}$ R. Dashen, W. H. Munk, K. M. Watson, and F. Zachariasen, Sound Transmission Through a Fluctuating Ocean, edited S. M. Flatté (Cambridge University Press, Cambridge, UK, 1979), 299 pp.

${ }^{10}$ A. K. Morozov and J. A. Colosi, "Entropy and scintillation analysis of acoustical beam propagation through ocean internal waves," J. Acoust. Soc. Am. 117(3), 1611-1623 (2005).

${ }^{11}$ A. K. Morozov and J. A. Colosi, "Stochastic differential equation analysis for sound scattering by random internal waves in the ocean," Acoust. Phys. 53(3), 335-347 (2007).

${ }^{12}$ L. M. Brekhovskih, V. V. Goncharov, and V. M. Kurtepov, "Weakly divergent bundles of sound rays in the Arctic," Atmos. Ocean. Phys. 31(3), 441-446 (1995).

${ }^{13}$ N. G. Van Kampen, "A cumulant expansion for stochastic differential equations," Physica 74, 215-247 (1974).

${ }^{14}$ C. E. Shannon, "A mathematical theory of communication," Bell Syst. Tech. J. 27(3), 379-423 (1948).

${ }^{15}$ C. E. Shannon, "A mathematical theory of communication," Bell Syst. Tech. J. 27(4), 623-656 (1948).

${ }^{16}$ P. R. Halmos, Measure Theory (Van Nostrand, Princeton, NJ, 1950), 304 pp.

${ }^{17}$ V. V. Goncharov and V. M. Kurtepov, "Formation and propagation of weakly diverging bundles of rays in a horizontally inhomogeneous ocean," Acoust. Phys. 40(5), 685-692 (1994).

${ }^{18}$ Yu. V. Petukhov, "A sound beam with minimal wave front divergence in a stratified ocean waveguide," Acoust. Phys. 40(1), 97-105 (1994).

${ }^{19}$ G. Casati, "Quantum chaos," Chaos 6(3), 391-398 (1996).

${ }^{20}$ V. Latora and M. Baranger, "Kolmogorov-Sinai entropy rate versus physical entropy," Phys. Rev. Lett. 82(3), 520-523 (1999).

${ }^{21}$ P. Gaspard and G. Nicolis, "Transport properties, Lyapunov exponents, and entropy per unit time," Phys. Rev. Lett. 65(14), 1693-1696 (1990).

${ }^{22}$ F. D. Tappert and X. Tang, "Ray chaos and eigenrays," J. Acoust. Soc. Am. 99, 185-195 (1996).

${ }^{23}$ M. A. Wolfson and S. Tomsovic, "On the stability of long-range sound propagation through a structured ocean," J. Acoust. Soc. Am. 109(6), 2693-2703 (2001). 